JOURNAL DE PHYSIQUE IV

Colloque C7, supplément au Journal de Physique III, Vol. 1, décembre 1991

C $7-3$

\title{
PROPERTIES AND MEDICAL APPLICATIONS OF NEAR-IR SOLID-STATE LASERS
}

B. STRUVE and G. HUBER*

LASERTEC HAMBURG GMBH, Stresemannstr. 364, D-2000 Hamburg 50, Germañy'

"Institut für Angewandte Physik, Universität Hamburg, Jungiusstr. 11, D-2000 Hamburg 36,

Germany

Abstract: $\quad$ Cr-Tm-YAG, Cr-Tm-Ho-YAG, and Er-YAG lasers at $2.01 \mu \mathrm{m}, 2.12 \mu \mathrm{m}$, and $2.94 \mu \mathrm{m}$, resp., are compared regarding single-shot and high-repetition rate efficiency. The $2 \mu \mathrm{m}$ lasers are intrinsically limited at higher operation temperatures, the Er-laser only shows thermal lensing. Thin $(3 \mathrm{~mm}) \mathrm{Tm}$ and Ho-laser rods allow high repetition rates $(30 \mathrm{~Hz})$ and with increasing rod diameters pulse energies up to $17 \mathrm{~J}$ are obtained.

\section{Introduction}

Flashlamp-pumped near-IR solid-state lasers based on $\operatorname{Tm}^{3+}|1|, \mathrm{Ho}^{3+}|2,3,4|$, and $\mathrm{Er}^{3+}|5|$ doped YAG have the potential for many applications, especially in medicine. However, the operation characteristics of the laser ions require special optimization of the laser set-up for different applications. We present an overview of the laser performances of Tm-, Hoand Er-lasers that gives data necessary for this optimization. 


\section{Cr-Tm-YAG, Cr-Tm-Ho-YAG Lasers}

The $\mathrm{Cr}$-Tm-laser emits at $2.01 \mu \mathrm{m}$, the $\mathrm{Cr}$-Tm-Ho-laser at $2.12 \mu \mathrm{m}$. The $\mathrm{Cr}$ ion acts as a broad band absorber of the flashlamp continuum radiation and effectively transfers the energy to the Tm ions. A cross-relaxation process results in two Tm ions excited into the

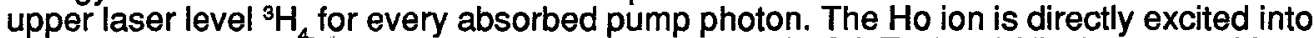
the upper laser level ${ }^{5} \mathrm{I}$ by non-radiative transfer from the ${ }^{3} \mathrm{H}_{4} \mathrm{Tm}$ level. The laser transitions for Tm and Ho terminate in the highest levels of the ground state multipletts, thus both are "quasi-3-level" lasers.

Typical single-shot efficiencies are given in Fig. 1 for a close-coupled single-lamp ceramical reflector. The behaviour for higher repetition rates (Fig. 2) shows a temperature dependence of the laser output for Ho and Tm. Increasing the rod temperature by higher mean pump powers decreases the gain by higher reabsorption from the thermally populated lower laser level and by lower Boltzmann population of the upper laser level. For the Ho-laser, in addition, the stored energy distribution between the Tm and Ho ions is shifted towards Tm decreasing the Ho-laser efficiency.

Optimization for high repetition rates requires thin laser rods: with $2.8 \mathrm{~mm}$ diameter $\mathrm{Cr}, \mathrm{Tm}, \mathrm{Ho}$-rods $30 \mathrm{~Hz}$ operation is possible |6|. On the other hand, single-shot high output energies up to $17 \mathrm{~J}$ are obtained with $6 \mathrm{~mm}$ diameter $\mathrm{Cr}, \mathrm{Tm}$, Ho-rods $|4|$.

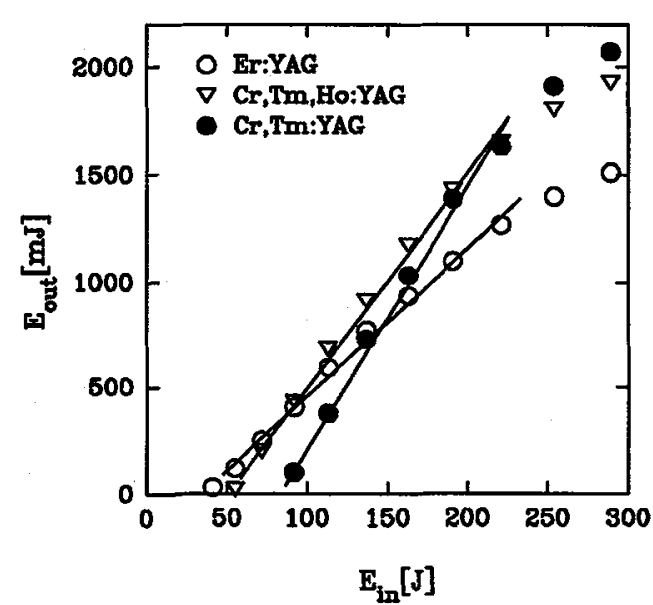

Fig. 1.: Single-shot operation of $\mathrm{Cr}-\mathrm{Tm}$ - and Cr-Tm-Ho-YAG, $3 \mathrm{~Hz}$-operation of Er-YAG (rods $4 X 80 \mathrm{~mm}$ without AR-coating, pulselength $500 \mu \mathrm{s}$, $\mathrm{T}=10 \%(\mathrm{Ho}, \mathrm{Er}), 20 \%(\mathrm{Tm}))$

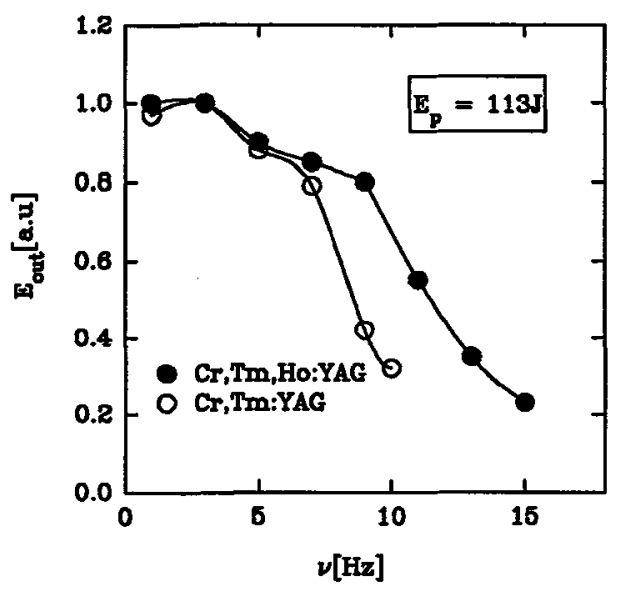

Fig. 2.: Relative pulse energies of Cr-Tm- and Cr-Tm-Ho-YAG for variable repetition rates (parameters as in Fig. 1.) 


\section{Er-YAG Laser}

The $2.9 \mu \mathrm{m}$ laser operates between the ${ }^{4} I_{1 / 2}$ and ${ }^{4} I_{13 / 2}$ levels being a 4-level-system. The efficiency is lower than for the Tm- and Ho-lasers because of the longer emission wavelength and a missing dominant cross-relaxation excitation process as in $\mathrm{Tm}$. The 4level-system does not have an intrinsic temperature dependent efficiency. The observed decrease of the output energies at higher repetition rates (Fig. 3 ) is ascribed to thermal lensing, because applying AR coatings to the endfaces of the laser rod cancels this effect.

By inserting two $\mathrm{CaF}_{2}$-prisms into the resonator the emission can be tuned to six lines in the long-wavelength part of the ${ }^{4} I_{1 / 2}->{ }^{4} I_{13 / 2}$ fluorescence: $2.80,2.83,2.86,2.87,2.90$, and $2.94 \mu \mathrm{m}$. Shorter wavelengths do not oscillate, because the corresponding transitions terminate in the lower states of the ${ }^{4} l_{132}$ multiplett which are populated by the long lifetime of this level. The $2.83 \mu \mathrm{m}$ and $2.94 \mu \mathrm{m}$ lines have comparable efficiencies, the others are 3 - 10 times weaker.

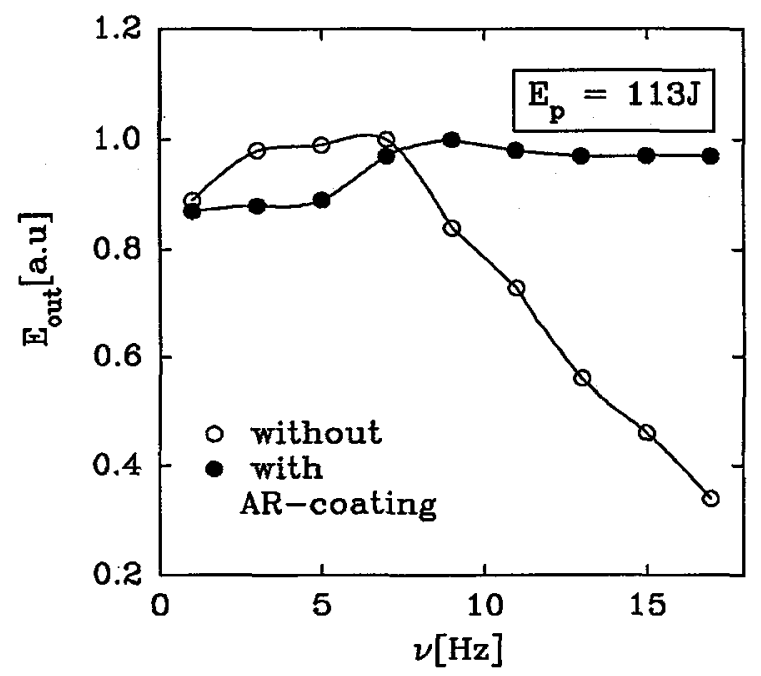

Fig. 3.:

Relative pulse energies of Er-YAG for variable repetition rates and rods with / without AR-coating (parameters as in Fig. $1, E_{\text {out }}=1.0$ corresponds to an output energy of $600 \mathrm{~mJ}$.

\section{Medical Applications}

With their large range of available parameters the near-IR lasers offer the possibility of being optimized regarding wavelength, pulse length, repetition rate, and pulse energy. As biological tissue contains, in general, a large amount of water, the absorption length in water at a specific wavelength determines the interaction: volume coagulation or ablation and cutting for large or small penetration depths, resp. The $2 \mu \mathrm{m} \mathrm{Tm}$-and Ho-lasers penetrate several tenths of a $\mathrm{mm}$ and are therefore suitable for ablation of membranes or other tissues with comparable dimensions. Synovectomy, e.g., may be performed endoscopically and ablation of inflamed synovia by $2 \mu \mathrm{m}$ laser radiation reduces bleeding and necrosis compared to standard mechanical techniques $|7|$.

The short penetration depth of the Er-laser of a few $\mu \mathrm{m}$ allows efficient ablation of dental hard substances without too much heating of the surrounding tissues. Shorter wavelength lasers (Tm, Ho, Nd) penetrate much deeper and do not allow localized ablation. To reach the ablation threshold high amounts of total energy have to be deposited that severely damage the tissue. 


\section{Conclusion}

Cr-Tm- and Cr-Tm-Ho-YAG lasers have been developed for high pulse energy (17 J) or high repetition rates $(30 \mathrm{~Hz}$ ) by optimizing the laser configuration, but allow also set-ups with $10 \mathrm{~W}$ of mean output power at $10 \mathrm{~Hz}$. In contrast, the Er-laser is not restricted regarding high repetition rates if $A R$-coated rods are used: more than $20 \mathrm{~Hz}$ are achieved without decrease in pulse energy.

\section{References}

|1| Quarles, G.S., Rosenbaum, A, Marquardt, C.C., Esterowitz, L, Optics Lett. 15 (1990) 42

|2| Antipenko, B.M., Glebov, A.S., Kiseleva, T.I., Pismennyi, V.A., Sov. Tech. Phys. Lett. 11 (1985) 284

|3| Duczynski, E.W., Huber, G., Ostroumov, V.G., Shcherbakov, I.A., Appl. Phys. Lett. 48 (1986) 1562

44 Teichmann, H.-O., Duczynski, E.W., Huber, G., Proc. SPIE 1021 (1989) 74

5) Zharikov, E.V., Zhekov, V.I., Kulevskii, L.A., Murina, T.M., Osiko, V.V., Prokhorov, A.M., Savel'ev, A.D., Smirnov, V.V., Starikov, B.P., Timoshenko, M.I.,

Sov. J. Quantum Electron. 4 (1975) 1039

16| Becker, T., Huber, G., v.d. Heide, H.-J., Mitzscherlich, P., Struve, B., Duczynski, E.W., Opt. Commun. 80 (1990) 47

|7| Möller, K.O., Lind, B., Schramm, V., Baretton, G., Hohlbach, G., $11^{\text {th }}$ Meet. Am. Soc. for Laser Med. and Surg. (San Diego, 1991)

\section{Note}

Parts of this work have been supported by the German Minister of Research and Technology (BMFT 13 N5803 and 13N5804.) 